\title{
Mapping stable direct and retrograde orbits around the triple system of asteroids (45) Eugenia
}

\author{
R. A. N. Araujo, ${ }^{1,2,3 \star}$ R. V. Moraes, ${ }^{2}$ A. F. B. A. Prado ${ }^{1}$ and O. C. Winter ${ }^{3}$ \\ ${ }^{1}$ National Institute for Space Research (INPE), CEP 12201-970, São José dos Campos, SP, Brazil \\ ${ }^{2}$ Instituto de Ciência e Tecnologia (ICT), Federal University de São Paulo (UNIFESP), CEP 12 247-014, São José dos Campos, SP, Brazil \\ ${ }^{3}$ Universidade Estadual Paulista (UNESP), Grupo de Dinâmica Orbital e Planetologia, CEP 12516-410, Guaratinguetá, SP, Brazil
}

Accepted 2017 August 23. Received 2017 August 23; in original form 2017 April 10

\begin{abstract}
It is widely accepted that knowing the composition and the orbital evolution of asteroids might help us to understand the process of formation of the Solar system. It is also known that asteroids can represent a threat to our planet. Such an important role has made space missions to asteroids a very popular topic in current astrodynamics and astronomy studies. Taking into account the increasing interest in space missions to asteroids, especially to multiple systems, we present a study that aims to characterize the stable and unstable regions around the triple system of asteroids (45) Eugenia. The goal is to characterize the unstable and stable regions of this system and to make a comparison with the system 2001 SN263, which is the target of the Advanced Spaceborne Thermal Emission and Reflection Radiometer (ASTER) mission. A new concept was used for mapping orbits, by considering the disturbance received by the spacecraft from all perturbing forces individually. This method has also been applied to (45) Eugenia. We present the stable and unstable regions for particles with relative inclination between $0^{\circ}$ and $180^{\circ}$. We found that (45) Eugenia presents larger stable regions for both prograde and retrograde cases. This is mainly because the satellites of this system are small when compared to the primary body, and because they are not close to each other. We also present a comparison between these two triple systems, and we discuss how these results can guide us in the planning of future missions.
\end{abstract}

Key words: methods: numerical - celestial mechanics - minor planets, asteroids: individual: (45) Eugenia-planets and satellites: dynamical evolution and stability.

\section{INTRODUCTION}

Knowledge of the composition and the dynamics of asteroids might help us to understand the process of formation of the Solar system and, thus, the formation and composition of our own planet. It is also known that asteroids can present a threat to our planet. These are some of the reasons why space missions that aim to visit asteroids in the Solar system are very popular topics in current astrodynamics and astronomy studies.

Examples of some successful missions are the Origins, Spectral Interpretation, Resource Identification, Security, Regolith Explorer (OSIRIS-REx; NASA), launched in 2016 September, which will return to Earth with a sample of the near-Earth asteroid (NEA), (101955) Bennu (Hergenrother et al. 2014), or the Hayabusa mission of the Japan Aerospace Exploration Agency (JAXA), launched in 2003 May, which explored the NEA, (25413) Itokawa (Yoshikawa et al. 2006), and returned to Earth in 2010 June with samples from the surface of the asteroid (Fujita et al. 2011).

Multiple systems of asteroids are interesting targets for space missions, as they increase the range of possible scientific investigations. This advantage was taken into account in the development of the Advanced Spaceborne Thermal Emission and Reflection Radiometer (ASTER) mission, which is the First Brazilian Deep Space Mission (Sukhanov et al. 2010). In this sense, the search for stable orbits around these bodies is very important, in terms of both science and engineering. Regions of stable orbits might be places full of dust and/or small pieces of materials, as well as possible locations for other members of the system. In a mission, they might also indicate good regions to place a spacecraft to observe the system.

The primary target of the ASTER mission is the NEA, 2001 SN263, which is a triple system of asteroids. The announcement of this mission has motivated studies aimed at characterizing the regions of stability of this system. Araujo et al. (2012) and Araujo, Winter \& Prado (2015) characterized stable and unstable regions around the components of this triple system, for the prograde and retrograde cases, through numerical integrations of the gravitational 
Table 1. Physical data and orbital elements of the components of the triple system (45) Eugenia (from Marchis et al. 2010 and Chamberlin et al. 2017, for the epoch JD 245 2980.0). The inclinations $i$ of Petit-Prince and Princesse are relative to the equator of Eugenia and the inclination of Eugenia is relative to the heliocentric ecliptic.

\begin{tabular}{lccccccc}
\hline Body & Orbits & $a$ & $e$ & $i$ & $\begin{array}{c}\text { Radius } \\
(\mathrm{km})\end{array}$ & \multicolumn{1}{c}{$\begin{array}{c}\text { Mass } \\
(\mathrm{kg})\end{array}$} \\
\hline Eugenia & Sun & $2.72 \mathrm{au}$ & 0.083 & 18.2 & $108.5^{a}$ & $5.63 \times 10^{18}$ \\
Princesse & Eugenia & $610.8 \mathrm{~km}$ & 0.069 & $18^{\circ}$ & 2.5 & $2.5 \times 10^{14}$ \\
Petit-Prince & Eugenia & $1164.5 \mathrm{~km}$ & 0.006 & $9^{\circ}$ & 3.5 & $2.5 \times 10^{14}$ \\
\hline
\end{tabular}

Note. ${ }^{a}$ Equivalent radius (Marchis et al. 2010).

$N$-body problem. Prado (2014) mapped orbits for a small body orbiting the asteroid $2001 \mathrm{SN} 263$. He considered the disturbance received from all the perturbing forces individually. This study used a new concept for mapping orbits that shows the relative importance of each force for a given orbit in the system. Such information has made it easier to decide which forces need to be included in a model for a given accuracy and nominal orbit.

Thus, in the present paper, we use a combination of both methods, presented in Araujo et al. (2012, 2015) and Prado (2014), in order to search for direct and retrograde stable orbits in the (45) Eugenia triple system of asteroids. (45) Eugenia is a triple system of asteroids of the Main Belt Asteroids (MBAs). It is composed by the central body Eugenia and by the satellites Petit-Prince and Princesse. The physical characteristics and orbital elements of this system are presented in Table 1. The physical and orbital data for Eugenia and its satellites were obtained from Marchis et al. (2010). Beauvalet \& Marchis (2014) updated these data with minor corrections of the order of the error bar of Marchis et al. (2010). Although these corrections must be important from an observational point of view, they do not significantly affect our long-term analysis of the stability of the particles within the system.

In particular, we searched for regions where direct orbits are unstable, but retrograde orbits are stable. Those orbits are very good for a potential mission. The probe can benefit from the stability of the orbit and thus minimize station-keeping manoeuvres. At the same time, it can travel in regions that are expected to be free of dust, so reducing the risk of collisions with natural debris. This idea has been proposed previously in the literature for the system 2001 SN263 (Araujo et al. 2015), and has now been extended to the (45) Eugenia system.

The main reason for this extension is that these two systems are completely different from each other in terms of physical characteristics. The system $2001 \mathrm{SN} 263$ is much smaller in size and distance, with the bodies very close to each other. This means that the possible orbits for the spatial exploration of the system are strongly perturbed, and stable orbits are very rare in many important regions. The system (45) Eugenia has components located at much larger distances, so the general mappings of the stable orbits are very different. Even the Kozai effects are reduced, allowing inclined orbits in many situations where they did not appear in 2001 SN263. It is easy to quantity the differences, in terms of the perturbation received by a small body in both systems, using the integral of the perturbing forces for one orbital period (Prado 2013).

The approach used here to find stable orbits is similar to the one used in the previous studies related to the 2001 SN263 system, and it is described in Section 2

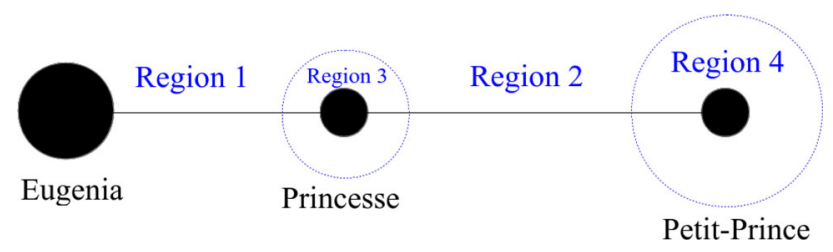

Figure 1. Representation of the triple system 45 Eugenia and the regions of influence of each component.

\section{METHOD}

The method adopted consisted of dividing the regions around the triple system in four distinct internal regions: two regions around the two small satellites of the system and the regions between the orbits of the two satellites (see Fig. 1).

Regions 3 and 4 around the satellites Princesse and Petit-Prince were defined by the Hill radius (Murray \& Dermott 1999), considering an approximation given by the two-body problem between Eugenia and each of the satellites separately. We found a region of about $29 \mathrm{~km}$ where the gravitational perturbation from Petit-Prince is dominant over the other bodies of the system. For Princesse, this region has a radius of about $15 \mathrm{~km}$. These values have guided us to define how the particles would be distributed within the system. This is better described in Sections 3.1-3.4

The $N$-body gravitational problem was integrated in time using the Gauss-Radau numerical integrator (Everhart 1985) for a timespan of $2 \mathrm{yr}$. Numerical integrations were performed considering a system composed of seven bodies, the Sun, the planets Earth, Mars and Jupiter, and the three components of the asteroid system, and thousands of particles randomly distributed around these components, including planar and inclined prograde and retrograde orbits.

Regarding the shape of the body, following Araujo et al. (2012, 2015), we considered as a first approximation the oblateness of Eugenia through the $J_{2}$ value. According to Marchis et al. (2010), the $J_{2}$ value for Eugenia is equal to $0.060 \pm 0.002$. Beauvalet \& Marchis (2014) fitted a dynamical model to simulate the observational data and to determine constraints in the dynamical parameter. From this fit, they found a $J_{2}$ value equal to $0.0589 \pm 0.0004$. Based on these values and on their error bar, in the integrations we considered $J_{2}=0.06$. The difference is less than 2 per cent, which does not significantly affect our long-term analysis of the stability of particles within the system.

The results are expressed by plots showing the percentage of particles that survived, for each set of initial conditions, as a function of the semimajor axis, eccentricity and inclination of the initial orbit. The regions where all the particles survived along the time-span of $2 \mathrm{yr}$ are called stable regions, whereas the regions where no particles survived along this time-span are called unstable regions.

In Section 3, we present the results of the numerical integrations for each region and also their implication in a space mission, making a comparison with the results previously found for the triple system 2001 SN263 in the context of the ASTER mission.

\section{RESULTS}

A first look at both systems of asteroids indicates that they are different in physical terms. The (45) Eugenia system has a central body that is much more massive with respect to its satellite bodies, when compared with the 2001 SN263 system. In this system, the three bodies have a more uniform distribution of masses among 
the bodies. The distances are also different, with the bodies of 2001 SN263 being much closer to each other than the bodies that compose the (45) Eugenia system. This combination of factors has a large effect on the stability of the orbits, with important consequences for selecting possible orbits for a potential mission that has this triple system as a target. These situations will be explored in detail when showing the results obtained here.

A preliminary general study of these differences can be carried out using the integral of the perturbing forces acting in this system (Prado 2013; Carvalho, Moraes \& Prado 2014; Oliveira \& Prado 2014; Oliveira, Prado \& Misra 2014; Sanchez, Prado \& Yokoyama 2014; Santos et al. 2015; Lara 2016; Sanchez, Howell \& Prado 2016; Short et al. 2017).

Basically, an integral of the perturbing forces acting in a small body within a given system is made over one orbital period. The idea is to give a first approximation of the differences of the perturbation level in both systems. Prado (2014) carried out this study for the 2001 SN263 system. Prado (2014) shows that the perturbation coming from the two smaller members of the system is several orders of magnitude larger than the other perturbations, so it is valid to make a first analysis of the perturbations using only those forces. Prado (2014) also describes the level of perturbation well, showing two peaks near the orbits of the smaller bodies. This result is expected as the perturbation increases when the small body comes closer to the perturbing bodies, but this figure quantifies the level of perturbation. It is noted that the magnitude of the perturbation is of the order of $0.025 \mathrm{~m} \mathrm{~s}^{-1}$ in the regions between the orbits of the two smaller bodies.

In this paper, we make a similar analysis for (45) Eugenia, the results of which are shown in Fig. 2. Because the idea is to measure the effects of the perturbations with respect to the gravity field of the main body, which is the most important comparison, a new type of integral is used. This index is calculated using the integration of the perturbing forces, which acts in a specific orbit divided by the gravity field of the main body, over one orbital period of the given orbit. The index used in Prado (2014) was calculated to compare different forces in the same system, and for orbits around the main body, so the effects of dividing the perturbation by the gravity field of the main body are small. However, in the present case, where the idea is to compare two different systems, as well as orbits around the main body and the two smaller bodies, it is very important to quantify the level of perturbations compared to the gravity field of the main body.

Fig. 2(a) considers orbits around the main body of the system, Eugenia, perturbed by the two natural satellites of the system, Princesse and Petit Prince. Note that there are two similar peaks near the orbits of the perturbing bodies as in 2001 SN263, as expected, but the magnitudes of the perturbation levels in all the regions are smaller, of the order of $10^{-8} \mathrm{~m} \mathrm{~s}^{-1}$ in the regions between both smaller bodies, and even smaller after the orbit of the exterior natural satellite. The peaks are not shown because we want to keep a better scale, to see more detail, but they are of the order of $10^{-6}$ $\mathrm{m} \mathrm{s}^{-1}$. The same type of integral index was calculated for 2001 SN263, because Prado (2014) uses a slight different index, showing values of the order of $10^{-6} \mathrm{~m} \mathrm{~s}^{-1}$ for the minimum points and near $10^{-5} \mathrm{~m} \mathrm{~s}^{-1}$ for the peaks. This clearly indicates the weaker effects of the perturbations, in the (45) Eugenia system, from the two companion bodies, for orbits around the main body of the system. This is a mathematical indication of much less perturbed orbits, which will have several consequences for the choice of locations to place a spacecraft to observe the system. It also shows the importance of using a scalar index to quantify the level of perturbation received in

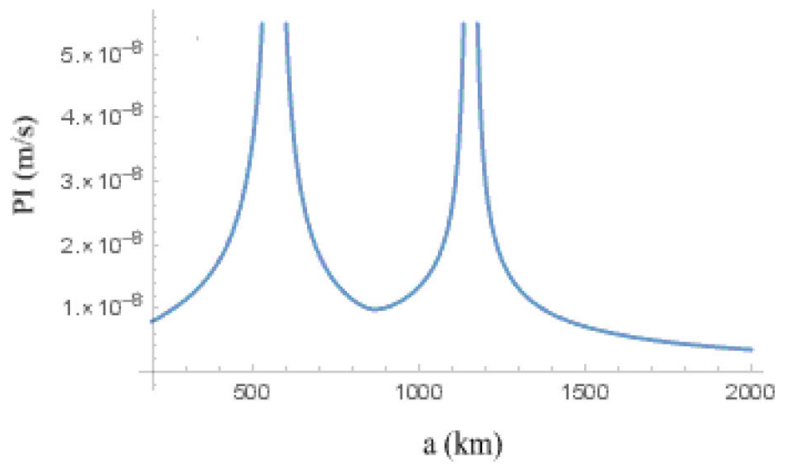

(a)

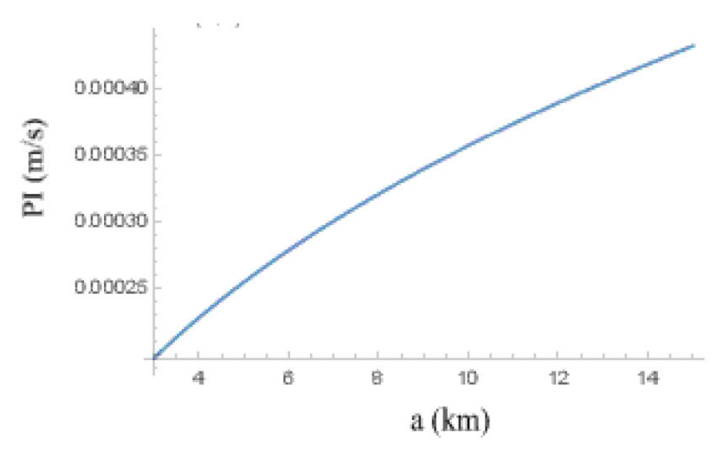

(b)

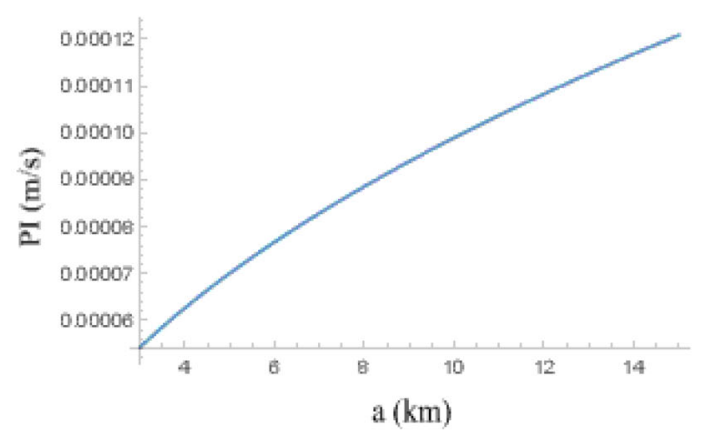

(c)

Figure 2. Perturbation integral $\left(\mathrm{m} \mathrm{s}^{-1}\right)$ as a function of the semimajor axis of the orbit of a small body within the triple system, considering perturbations coming from (a) Princesse and Petit Prince for circular orbits, (b) Eugenia and Petit-Prince and (c) Eugenia and Princesse.

a given orbit, so it is not necessary to limit the study to the expected general behaviour of the perturbation level, without a quantitative analysis of the forces.

The integral of the perturbing forces acting in the system give us an estimation of the stability of the system. It quantifies the perturbations due to the bodies of the system, indicating regions where particles or a small body would be more or less perturbed. Nevertheless, it does not take into account other dynamical effects such as mean motion resonances, the Kozai resonances for highinclined orbits or the known increase in the stability of retrograde orbits. The results presented in Fig. 2 show, as expected, that the perturbation from the satellites is small in the triple system Eugenia. 


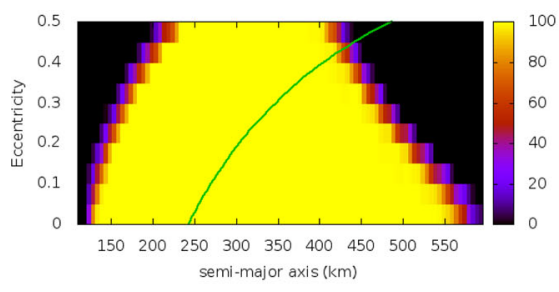

(a)

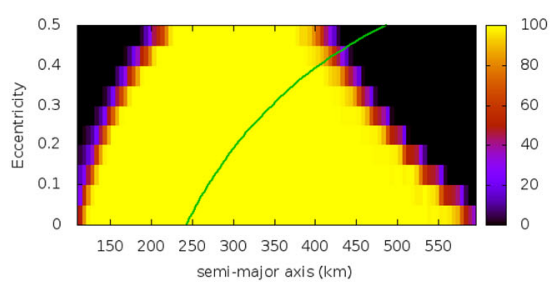

(d)

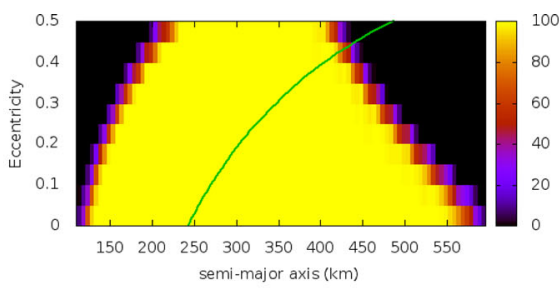

(b)

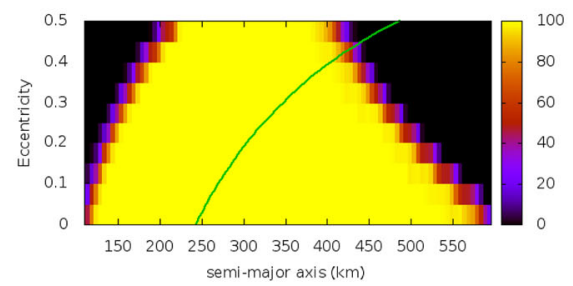

(e)

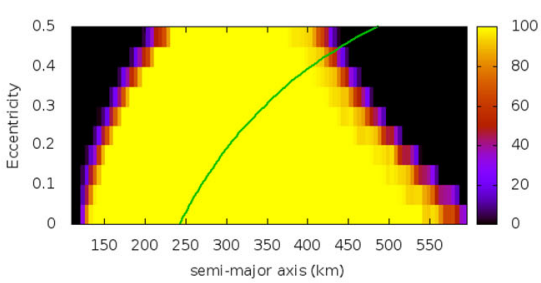

(g)

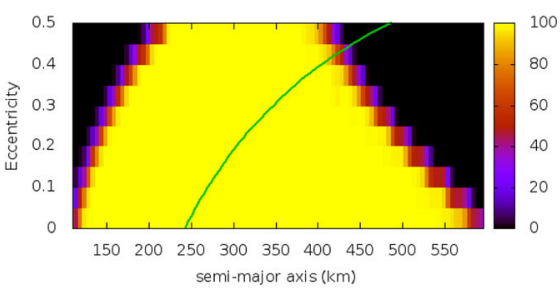

(c)

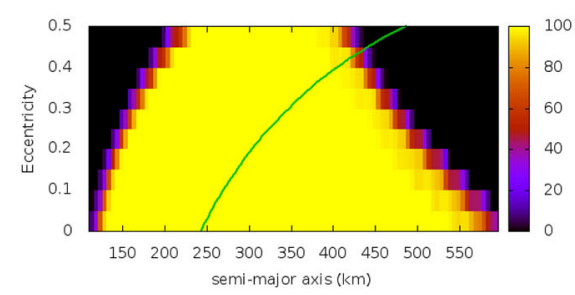

(f)

Figure 3. Plots of $a$ versus $e$ of the stability of region 1 for the triple system (45) Eugenia, for a time-span of 2 yr. In this region, the particles orbit Eugenia with orbital inclination: (a) $I=0^{\circ}$; (b) $I=30^{\circ}$; (c) $I=60^{\circ}$; (d) $I=90^{\circ}$; (e) $I=120^{\circ}$; (f) $I=150^{\circ}$; (g) $I=180^{\circ}$. The colour-coded scale indicates the percentage of particles that survive in this region, going from 0 per cent (instability) to 100 per cent (stability). As explained in the text, for a particle with a pericentric distance smaller than $243 \mathrm{~km}$ (on the left of the green solid curves) a model considering a more realistic shape of Eugenia must be adopted.

Thus, we expect the regions around the components of this system to be stable. In order to verify whether this system is subject to the perturbations cited above and how they differ from what is predicted by the integral method, we have performed numerical simulations of particles distributed within the system and we have verified their stability as a function of their initial orbit, as follows.

\subsection{Region 1}

Region 1 was defined as the region around Eugenia going from $110 \mathrm{~km}$ (1.01 radius of Eugenia) to $595 \mathrm{~km}$ (limited by the orbit of Princesse, considering its region of influence).

The particles were distributed within this region orbiting Eugenia with the following orbital elements: $110 \leq a \leq 595 \mathrm{~km}$, taken every $5 \mathrm{~km} ; 0 \leq e \leq 0.5$, taken every $0.05 ; 0^{\circ} \leq I \leq 180^{\circ}$, taken every $30^{\circ}$. For each combination of $a$ versus $e$, we considered 100 particles with random values of $f, \omega$ and $\Omega$, where $a$ is the semimajor axis, $e$ is the eccentricity, $I$ is the inclination, $f$ is the true anomaly, $\omega$ is the argument of the pericentre and $\Omega$ is the longitude of the ascending node. This combination of initial conditions resulted in a total of approximately 108000 particles distributed within region 1 .

The stability and instability are defined by the number of particles that survived (no ejections or collisions) throughout the numerical integrations. The collision was defined by the physical radius of the bodies. For region 1, an ejection was considered every time that the relative distance between a given particle and Eugenia was greater than $595 \mathrm{~km}$ (the limit of the region 1).

The results for region 1 are presented in Fig. 3. They show that approximately the whole region is stable. The instability appears only for the orbits really close to Eugenia or Princesse, or for those orbits with such high eccentricities that the crossing of orbits becomes possible, or for those orbits whose pericentre is inside Eugenia, leading to collisions.

The results also show that there are no effects from the inclination of the orbit on the survival of the particles. This is opposite to what was found in the system 2001 SN263, where the inclination played an important role in the evolution of the orbits of the particles. Araujo et al. (2012) showed, in their fig. 6, that the Kozai effect reduces the regions of stable orbits with an increase of the inclination, with a maximum effect at $60^{\circ}$ of inclination. Studying retrograde orbits, Araujo et al. (2015) showed, in their fig. 8, that there are locations in region 1 with stable retrograde and unstable direct orbits. These regions are excellent locations to place a space probe in order to observe the central body, as explained before.

Therefore, we do not have this option for selecting orbits in order to observe the main body of the (45) Eugenia system. This is because the third-body perturbation acting in a small body within this system is much smaller in (45) Eugenia, when compared to the 2001 SN263 system. There are two reasons for this. The third-body perturbation depends on the mass ratio between 


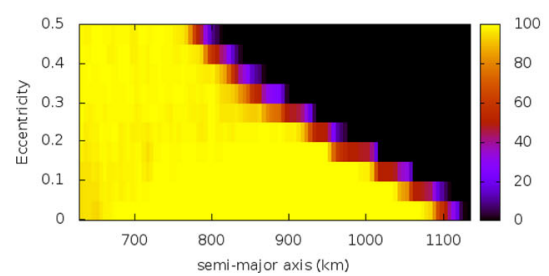

(a)

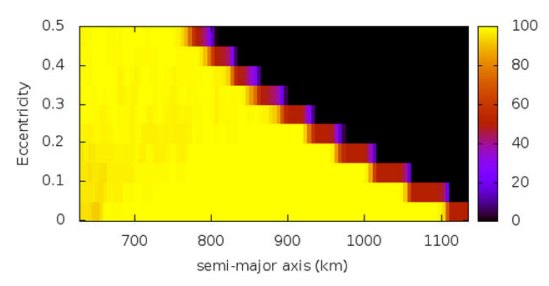

(d)

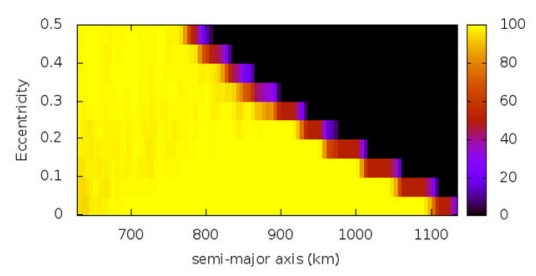

(b)

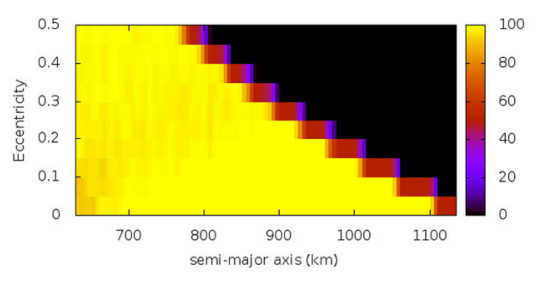

(e)

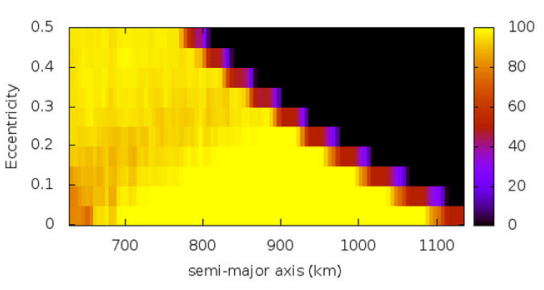

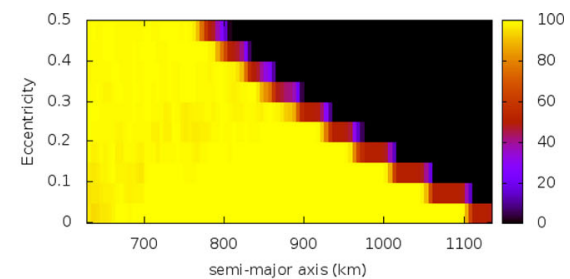

(c)

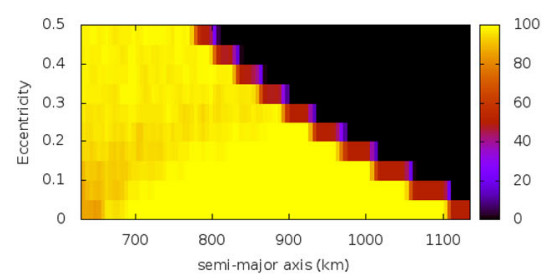

(f)

(g)

Figure 4. Plots of $a$ versus $e$ of the stability of region 2 for the triple system (45) Eugenia, for a time-span of 2 yr. In this region, the particles orbit Eugenia with orbital inclinations: (a) $I=0^{\circ}$; (b) $I=30^{\circ}$; (c) $I=60^{\circ}$; (d) $I=90^{\circ}$; (e) $I=120^{\circ}$; (f) $I=150^{\circ}$; (g) $I=180^{\circ}$. The colour-coded scale indicates the percentage of particles that survive in this region, going from 0 per cent (instability) to 100 per cent (stability).

the perturbing and the central body. This ratio is (the mass of Princesse, the closest perturbing body $) /($ the mass of Eugenia) $=$ $\left(2.51 \times 10^{14}\right) /\left(5.63 \times 10^{18}\right)=4.46 \times 10^{-5}$ in the case of the (45) Eugenia system and $1.06 \times 10^{-2}$ for the 2001 SN263 system, which is a very large difference. Another reason is that both companion asteroids are located very far from the main body, more than $600 \mathrm{~km}$ for the closest one, as shown in Table 1. This distance is about $3.8 \mathrm{~km}$ in the $2001 \mathrm{SN} 263$ system. Thus, a combination of these two reasons means that the Kozai effects are much smaller and are not able to modify the evolution of planar and inclined orbits significantly.

The particles in region 1 are those that are affected by the irregular shape of Eugenia. Jiang et al. (2016) explored these effects on the two satellites Princesse and Petit-Prince. Nevertheless, Chanut, Winter \& Tsuchida (2014) has shown how the relative error on the gravitational potential depends on the radial distance of a particle relative to a central irregular-shaped body. In their work, the gravitational potential was determined for the asteroid 433 Eros, modelled using the polyhedral model. They compared the results obtained with a simpler model where Eros was considered a point of mass. Their results show an error smaller than 10 per cent at a distance of about 1.6 from the physical semimajor axis of the body. Applying this result to Eugenia, considering the physical semimajor axis of Eugenia to be $152 \mathrm{~km}$ (Jiang et al. 2016), we estimate that particles within a distance of about $243 \mathrm{~km}$ are those that be affected by the irregular shape of Eugenia. Thus, for a particle with a pericentric distance smaller than $243 \mathrm{~km}$ (on the left of the green solid curves shown in Fig. 3), a model considering a more realistic shape of Eugenia must be adopted. For the other regions, the differences are not significant.

\subsection{Region 2}

Region 2 is the region between the orbits of the two satellites, and it is filled with particles orbiting Eugenia. This region goes from $625.8 \mathrm{~km}$ (limited by the orbit of Princesse, considering its region of influence) to $1136.5 \mathrm{~km}$ (limited by the orbit of Petit-Prince, considering its region of influence). The particles are distributed within this region orbiting Eugenia with $630 \leq a \leq 1135 \mathrm{~km}$, taken every $5 \mathrm{~km}$. The other elements are taken to be the same as for region 1 . This combination of initial conditions resulted in a total of approximately 112000 particles distributed within region 2 .

The results for region 2 are presented in Fig. 4. As found for region 1, we see that approximately the whole region 2 is stable. The instability appears only for orbits that cross the orbit of PetitPrince. The results for this region also show that, once again, there are no effects from the inclination on the survival of the particles, as in region 1. Once more, this is different from what occurred in 2001 SN263, as detailed in fig. 7 of Araujo et al. (2012), where the inclination has a strong effect on the evolution of the orbits of the 


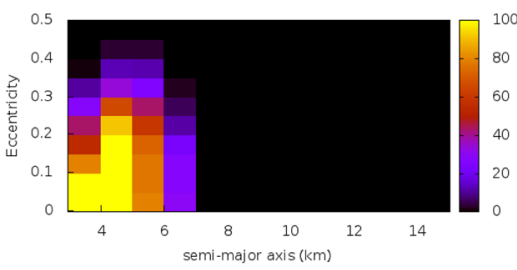

(a)

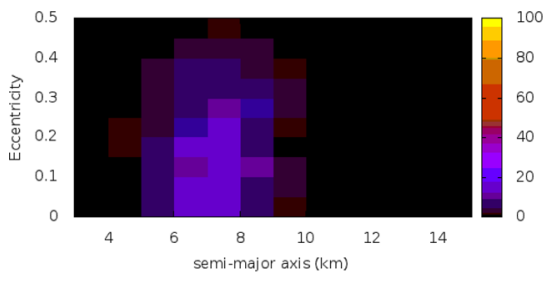

(d)

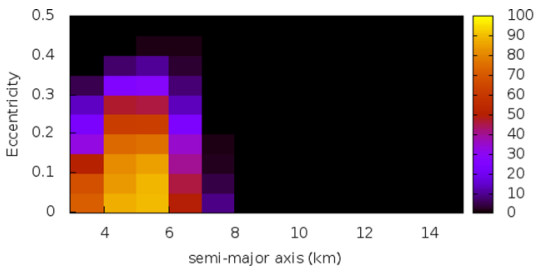

(b)

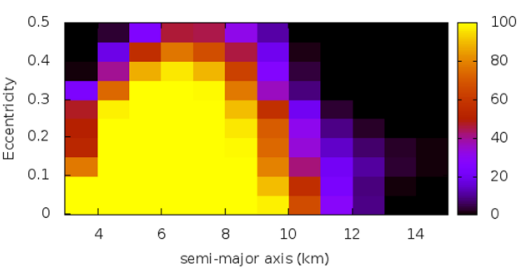

(e)

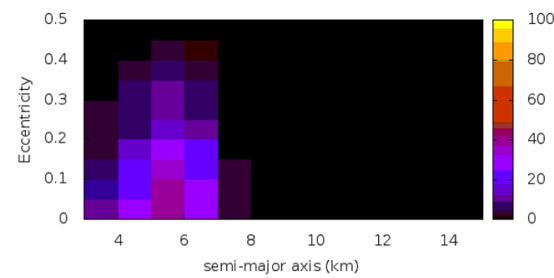

(c)

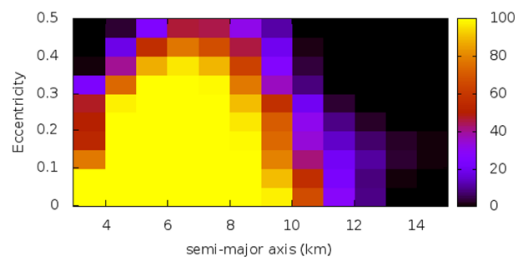

(f)

Figure 5. Plots of $a$ versus $e$ of the stability of region 3 for the triple system (45) Eugenia, for a time-span of 2 yr. In this region, the particles orbit Princesse with orbital inclinations: (a) $I=0^{\circ}$; (b) $I=30^{\circ}$; (c) $I=60^{\circ}$; (d) $I=120^{\circ}$; (e) $I=150^{\circ}$; (f) $I=180^{\circ}$. The colour-coded scale indicates the percentage of particles that survive in this region, going from 0 per cent (instability) to 100 per cent (stability). The diagram for $I=90^{\circ}$ is not presented, as all the particles in this region with this inclination were lost in $2 \mathrm{yr}$ (collisions or ejections).

particles, with a maximum at $75^{\circ}$. The study of retrograde orbits by Araujo et al. (2015) showed a very large region of stable/retrograde and unstable/direct orbits (see their fig. 8). This means that we do not have this option for selecting orbits for a space mission in this region for the (45) Eugenia system. The reason is the same as before (i.e. the weak Kozai effect due to the smaller mass ratio and larger distances of the third perturbing body).

\subsection{Region 3}

Region 3 is defined as the region around Princesse, going from $2.6 \mathrm{~km}$ (1.04 radius of Princesse) to $15 \mathrm{~km}$ (the Hill radius of Princesse with respect to Eugenia)

The particles are distributed within this region orbiting Princesse with $630 \leq a \leq 1135 \mathrm{~km}$, taken every $5 \mathrm{~km}$. The other elements are taken to be the same as before. This combination of initial conditions results in a total of approximately 14300 particles distributed within region 3 .

The results for region 3 are presented in Fig. 5. The results for this region show that there are strong effects of the inclination on the survival of the particles. The Kozai effect is now present because the third-body perturbation is now very strong as the mass ratio between the main body and the perturbing body is also $4.46 \times 10^{-5}$. Therefore, orbits with higher inclination are more stable than the planar orbits, when they are not in the region where the Kozai resonance action occurs. However, the results are still different from the results obtained for 2001 SN263, as shown in Araujo et al. (2012). For the system 2001 SN263, no stable orbits were found around the closer smaller body, because of the very strong perturbation coming from the central body. Even retrograde stable orbits do not exist, as shown in Araujo et al. (2015). This means that the (45) Eugenia system has excellent natural orbits for a space mission to observe the smaller body closer to the central body, in contrast to the 2001 SN263 system, where it is necessary to have orbital control in order to observe this body.
It is clear, from Fig. 5, that retrograde circular orbits with semimajor radius between 7 and $10 \mathrm{~km}$, depending on the initial eccentricity, are excellent for an exploration mission to this system, because they are stable while the equivalent direct orbits (same size and shape) are unstable.

\subsection{Region 4}

Region 4 is defined as the region around Petit-Prince, going from $3.6 \mathrm{~km}$ (1.03 radius of Petit-Prince) to $28 \mathrm{~km}$ (the Hill radius of Petit-Prince with respect to Eugenia)

The particles are distributed within this region orbiting PetitPrince with $3.6 \leq a \leq 28 \mathrm{~km}$, taken every $5 \mathrm{~km}$. The other elements were are taken to be the same as before. This combination of initial conditions results in a total of approximately 27500 particles distributed within region 4 .

The results for this region show smaller effects from the inclination in the survival of the particles, when compared to region 3 . The Kozai effect is present, but it is very small. Although the third body still has a larger mass, the distances between the bodies are much larger, compared to region 3. The results for 2001 SN263, shown in fig. 8 of Araujo et al. (2012), indicate much larger effects in the most distant body. This is because of the much smaller distances involved in the system 2001 SN263, which contributes to higher third-body perturbations. A study of the retrograde orbits showed larger regions of stable orbits, as for the system 2001 SN263, shown in fig. 8 of Araujo et al. (2015). This means that the (45) Eugenia system has excellent locations to place a spacecraft in order to observe the smaller body that is far away from to the central body.

The results for regions 1-4 show the existence of stable orbits in the regions around the three bodies of the system. These results are different from the results obtained when studying the 2001 SN263 system, where no stable orbits were found near the smallest body of the system. Several other important differences were found. In particular, there are no places around the main body where there are stable retrograde orbits and unstable prograde orbits. This means 


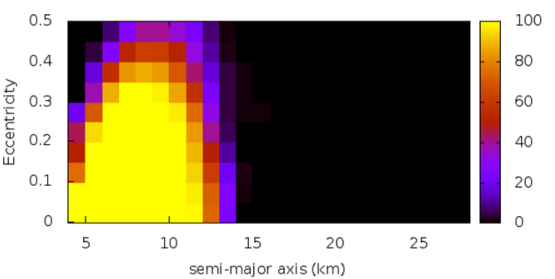

(a)

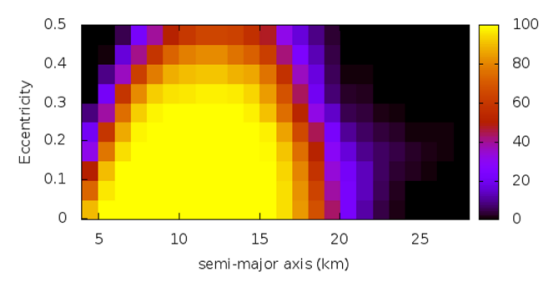

(d)

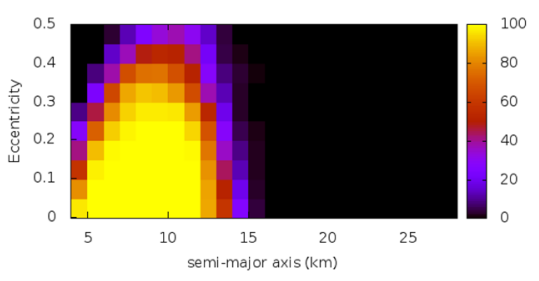

(b)

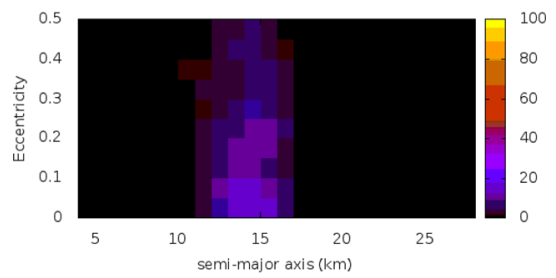

(c)

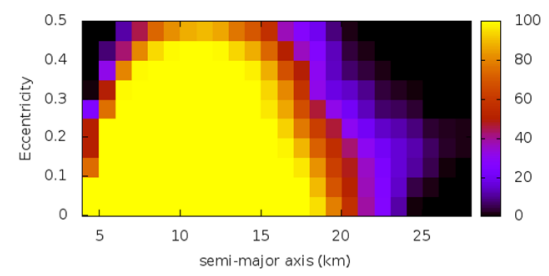

(e)

Figure 6. Plots of $a$ versus $e$ of the stability of region 4 for the triple system (45) Eugenia, for a time-span of 2 yr. In this region, the particles orbit Petit-Prince with orbital inclinations: (a) $I=0^{\circ}$; (b) $I=30^{\circ}$; (c) $I=120^{\circ}$; (d) $I=150^{\circ}$; (e) $I=180^{\circ}$. The colour-coded scale indicates the percentage of particles that survive in this region, going from 0 per cent (instability) to 100 per cent (stability). The diagrams for $I=60^{\circ}$ and $I=90^{\circ}$ are not presented, as all the particles in this region with this inclination were lost in $2 \mathrm{yr}$ (collisions or ejections).

that this strategy for choosing orbits cannot be used to observe the main body. However, similar situations are found around the smaller bodies of the system. In general, the lower perturbation level that occurs in all the regions of (45) Eugenia means that it is much easier to place a spacecraft in this system, from the point of view of longer-duration missions requiring less orbital control. This is a key point in deep space missions, because of the technical difficulties of carrying more fuel and making frequent orbital manoeuvres at this distance.

It is clear, from Fig. 6, that retrograde circular orbits with semimajor radius from 14 to $18 \mathrm{~km}$, depending on the initial eccentricity, are excellent for an exploration mission to this system, because they are stable and direct orbits of the same size are unstable.

\section{CONCLUSIONS}

The (45) Eugenia system has been studied in detail, with the goal of finding stable orbits in the several regions of this triple asteroid system. The stable orbits are important to indicate regions of possible accumulation of dust, or even good locations to look for a new member of the asteroid system. In that sense, stable regions have been found, as a function of the semimajor axis, eccentricity and inclination of the orbits, around all the bodies that compose the system.

In general, the results have shown that the (45) Eugenia system is much less perturbed than the triple system 2001 SN263, which was studied previously. The integral of the perturbing forces quantified this fact in numbers. In particular, the Kozai effect of destroying inclined orbits was not present in orbits around the central body, because of the small mass ratio and the large distance of the thirdbody perturbation. However, this effect is present in orbits around the two satellites.

These stable orbits are also important in terms of astronautical applications, because regions of space that have unstable/direct and stable/retrograde orbits are good locations to place a spacecraft, as they give stability for the orbit and there is a low risk of collisions with particles. A new type of integral index was defined, taking into account the perturbations coming from other bodies of the systems rather than the body that the spacecraft is orbiting. Using this, we can compare the perturbation levels, which indicates that orbits around the main body of the (45) Eugenia system are about $10^{4}$ times less perturbed than the equivalent orbits of the 2001 SN263 system. Regarding the companion bodies, the internal body of the (45) Eugenia system is also about $10^{4}$ times less perturbed than the equivalent body of the 2001 SN263 system. For the external bodies, the levels of perturbations are similar for both systems.

The results show that orbits in which a spacecraft can be located using the difference in the stability for direct and retrograde orbits do not exist in the (45) Eugenia system for regions near the central body and between the orbits of the two smaller bodies, as found for the 2001 SN263 system. In contrast, such regions have been found around the two smaller bodies of the system, covering regions where no stable orbits were found for the 2001 SN263 system. This means that, in terms of observing the smaller bodies of the system, the triple asteroid (45) Eugenia offers better locations to place a spacecraft, with cleaned stable retrograde orbits. The numerical results show that orbits with a semimajor radius from 7 to $10 \mathrm{~km}$ are very good choices for orbits around Princesse, and orbits with a semimajor radius from 14 to $18 \mathrm{~km}$ are very good choices for orbits around Petit-Prince.

\section{ACKNOWLEDGEMENTS}

This work was funded by $\mathrm{CNPq}$ Procs. 150378/2015-7 and 301338/2016-7, and by FAPESP Procs. 2016/24561-0 and 2011/08171-3. This support is gratefully acknowledged.

\section{REFERENCES}

Araujo R. A. N., Winter O. C., Prado A. F. B. A., Sukhanov A., 2012, MNRAS, 423, 3058

Araujo R. A. N., Winter O. C., Prado A. F. B. A., 2015, MNRAS, 449, 4404 
Beauvalet L., Marchis F., 2014, Icarus, 241, 13

Chamberlin A. et al., 2017, JPL's HORIZONS System, Available at: https://ssd.jpl.nasa.gov/horizons.cgi

Carvalho J. P. S., Moraes R. V., Prado A. F. B. A., 2014, Mathematical Problems in Engineering, 2014, 529716

Chanut T. G. G., Winter O. C., Tsuchida M., 2014, MNRAS, 438, 2672

Everhart E., 1985, in Carusi Carusi A., Valsecchi G. B., eds, Dynamics of Comets: Their Origin and Evolution. Reidel, Dordrecht, p. 185

Fujita K. et al., 2011, PASJ, 63, 961

Hergenrother C. W. et al., 2014, preprint (arXiv:1409.4704)

Jiang Y., Zhang Y., Baoyin H., Li J., 2016, Ap\&SS, 361, 306

Lara M., 2016, Journal of Guidance, Control, and Dynamics, 39, 2157

Marchis F. et al., 2010, Icarus, 210, 635

Murray D. C., Dermott S. F., 1999, Solar System Dynamics. Cambridge Univ. Press, Cambridge

Oliveira T. C., Prado A. F. B. A., 2014, Acta Astron., 104, 350

Oliveira T. C., Prado A. F. B. A., Misra A. K., 2014, in Wilson R. S., Zanetti R., Mackison D. L., Abdelkhalik O., eds, Proc. 24th AAS/AIAA Space Flight Mechanics Meeting 2014, Advances in the Astronautical Sciences Vol. 152. AAS Publications, San Diego, CA, p. 3081

Prado A. F. B. A., 2013, Mathematical Problems in Engineering, 2013, 415015
Prado A. F. B. A., 2014, Advances in Space Research, 53, 877

Sanchez D. M., Prado A. F. B. A., Yokoyama T., 2014, Advances in Space Research, 54, 1008

Sanchez D. M., Howell K. C., Prado A. F. B. A., 2016, in Zanetti R., Russell R. P., Ozimek M. T., Bowes A. L., eds, Proc. 26th AAS/AIAA Spaceflight Mechanics Meeting, Advances in the Astronautical Sciences Vol. 158. AAS Publications, San Diego, CA, p. 278

Santos J. C., Carvalho J. P. S., Prado A. F. B. A., Moraes R. V., 2015, J. Physics Conf. Series. 641, 012011

Short C., Howell K., Haapala A., Dichmann D., 2017, J. Astronaut. Sci., 64 , 156

Sukhanov A. A., Velho H. F. C., Macau E. E., Winter O. C., 2010, Cosmic Research, 48, 443

Yoshikawa M. et al., 2006, in Workshop on Spacecraft Reconnaissance of Asteroid and Comet Interiors, no. 3038 (Available at: http://www.lpi.usra.edu/meetings/recon2006/pdf/3038.pdf)

This paper has been typeset from a $\mathrm{T}_{\mathrm{E}} \mathrm{X} / \mathrm{LAT}_{\mathrm{E}} \mathrm{X}$ file prepared by the author. 